Rev Biomed 2001; 12:262-271.

\title{
Micoplasmas y su importancia médica.
}

Revisión

José A. Rivera-Tapia ${ }^{1}$, Ma. Lilia Cedillo-Ramírez¹, Mayra Vega-Benítez².

${ }^{1}$ Centro de Investigaciones en Ciencias Microbiológicas, ${ }^{2}$ Escuela de Biología, Instituto de Ciencias de la Benemérita Universidad Autónoma de Puebla, Puebla, Puebla, México.

\section{RESUMEN.}

En 1898 se describieron por primera vez a los microorganismos autorreplicables más pequeños, denominados hoy en día micoplasmas. Estos microorganismos exhiben características morfológicas, metabólicas y moleculares muy particulares; su importancia ha generado interés en el estudio de enfermedades humanas, ya que durante la práctica clínica diaria se relacionan con enfermedades tanto agudas como crónicas, que en ciertas ocasiones llegan a términos fatales.

Su distribución en hospederos es diversa y se encuentran en peces, reptiles, aves, mamíferos y en el humano. En este último se han aislado de diversos sitios anatómicos, tanto en individuos con diferentes padecimientos como en individuos sanos. Su presencia se ha observado en tracto respiratorio y genitourinario, en complicaciones neurológicas y en sangre. Donde ha llamado más la atención es su probable papel como cofactor en la progresión del síndrome de la inmunodeficiencia adquirida.
Hace ya más de veinte años se estudia el papel de Mycoplasma fermentans en diversas enfermedades, así como los mecanismos gracias a los cuales interaccionan con las células hospederas, como son la supervivencia en células fagocíticas, su replicación intracelular, la capacidad inmunomoduladora y especialmente su variación antigénica.

(Rev Biomed 2001; 12:262-271)

Palabras clave: Micoplasmas, hospederos, sitios de colonización, SIDA.

\section{SUMMARY.}

Mycoplasmas and their medical importance.

In 1898 mycoplasmas were described as the smallest self-replicating bacteria, these microorganisms exhibit special morphologic, metabolic and molecular characteristics. Their role in human diseases has generated interest since they

Solicitud de sobretiros: M. en C. José A. Rivera-Tapia. Laboratorio de Micoplasmas del Centro de Investigaciones Microbiológicas del Instituto de Ciencias de la Benemérita Universidad Autónoma de Puebla, Edificio 76 Complejo de Ciencias. Ciudad Universitaria. C.P. 72570, Puebla, Pue., México.E-mail: jart70@yahoo.com 


\section{JA Rivera-Tapia, ML Cedillo-Ramírez, M Vega-Benítez.}

are frequently related with chronic acute and even fatal diseases during the everyday clinical practice.

Their distribution in hosts is diverse, since they have been isolated from fish, reptiles, birds, mammals and humans. In this last host they have been isolated from diverse anatomical sites, in both healthy and sick subjects. Their presence has been observed in respiratory and genitourinary tract, neurological complications as well as in blood. Special attention has been paid to their possible relationship as a cofactor in the progression of the Acquired Immunodeficiency Syndrome. The role of Mycoplasma fermentans in several diseases has been studied during the last twenty years. Several studies are being carried out in order to determine its role as a pathogen and the mechanism it uses to interact with the hosts cells, to survive inside phagocytes cells, its immunomodulatory ability and especially its antigenic variation.

(Rev Biomed 2001; 12:262-271)

Key words: Mycoplasmas, AIDS.

\section{INTRODUCCIÓN.}

En el año de 1898 Nocard y Roux describieron a los micoplasmas, demostrando que la pleuroneumonía en ganado vacuno era causada por organismos que pasan filtros que retenían bacterias. Estos investigadores desarrollaron un medio de agar enriquecido que soportaba el crecimiento colonial de estos microorganismos. Las colonias de los micoplasmas (figura 1) no son iguales al resto de las bacterias, presentando un

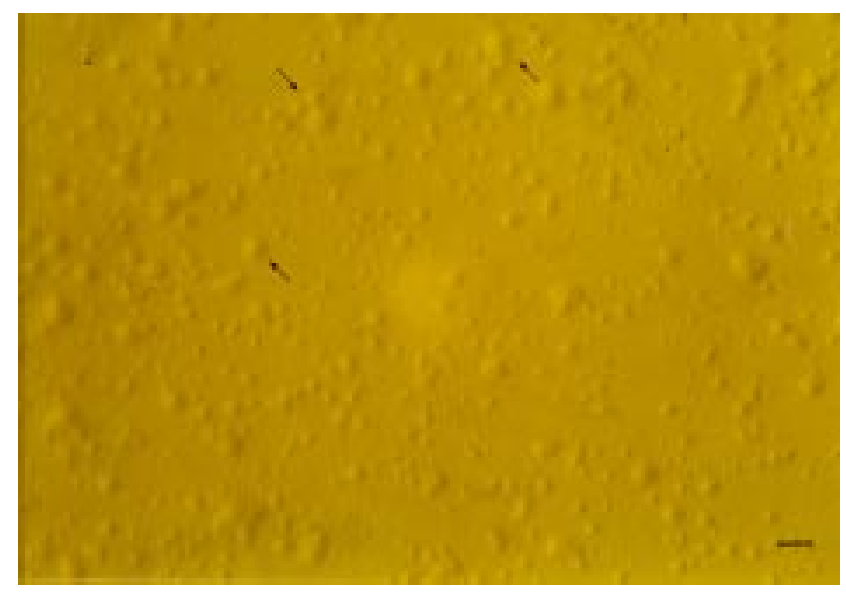

Figura 1.- Crecimiento colonial de Mycoplasma fermentans en agar Eaton. Las flechas indican las colonias donde se observan los rasgos característicos de colonias en forma de "huevo frito" (escala de la barra $=0.5 \mathrm{~mm}$ ).

centro denso debido al crecimiento dentro del agar $\mathrm{y}$ crecimiento poco denso en las zonas periféricas (1).

Los micoplasmas pertenecen a la Clase Mollicute (cuadro 1) y se caracterizan por carecer de pared celular o precursores químicos del peptidoglucano, presentando formas pleomórficas (figura 2), son filtrables a través de membranas de $450 \mathrm{nM}$, con requerimientos nutricionales exigentes y con un genoma que puede ir de las 577 a 2200 $\mathrm{kpb}(2)$.

La familia Mycoplasmataceae requiere de colesterol para su crecimiento, algunos hidrolizan urea, oxidan ácidos grasos de cadenas cortas a través de la beta-oxidasa o degradan azúcares durante sus procesos glucolíticos. Comúnmente infectan animales causando enfermedades y frecuentemente contaminan cultivos celulares (3).

Cuadro 1

Clasificación de los micoplasmas.

\begin{tabular}{llll}
\hline Clase & & Mollicutes & \\
Orden & & Mycoplasmatales & \\
Familia & Mycoplasmataceae & Acholeplasmateceae & Spiroplasmataceae \\
Género & Mycoplasma & Acholeplasma & Spiroplasma \\
& Ureaplasma & & \\
\hline
\end{tabular}


Micoplasmas y su importancia médica.

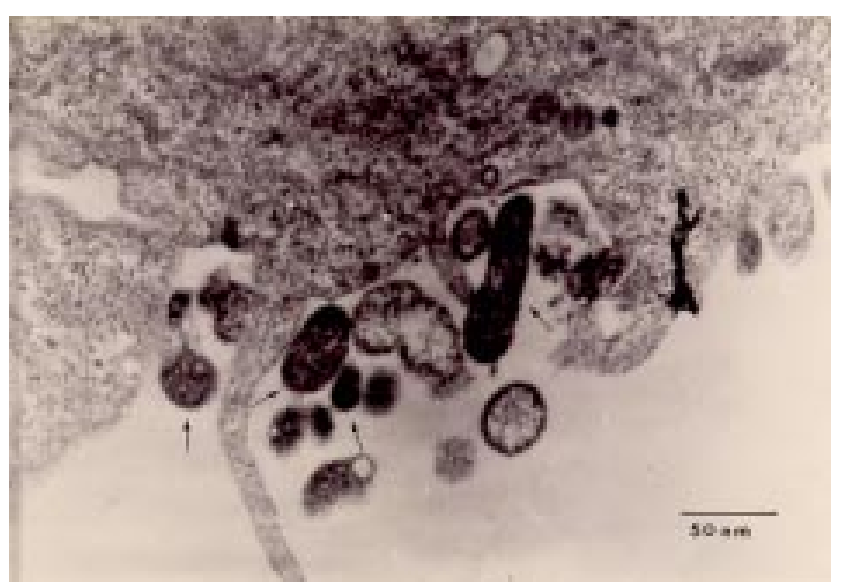

Figura 2.- Micrografía electrónica de transmisión que muestra una preparación de Mycoplasma fermentans (x 20000) adherida a células Hep-2. Las flechas indican las formas pleomórficas de los micoplasmas.

En 1962 se demostró que el agente etiológico de la neumonía atípica primaria era un micoplasma (Mycoplasma pneumoniae) (4). El papel de los micoplasmas en enfermedades humanas ha sido estudiado, especialmente en aparato respiratorio y genital, así como en enfermedades articulares (3). La presente revisión ofrece un panorama del papel que tienen los micoplasmas en relación a ciertas enfermedades de humanos, las cuales en ocasiones llegan a términos fatales, en relación a lo anterior se recomienda considerar más a estos microorganismos en la actividad clínica diaria y no descartarlos en padecimientos que no presenten un agente etiológico de rutina.

\section{Distribución de los Mollicutes.}

Se han descrito más de 150 especies en la Clase Mollicutes, incluyendo 92 especies del género micoplasmas en humano, mamíferos, aves, reptiles y peces $(5,6)$. Algunos micoplasmas que se presentan en animales han sido aislados de humanos en pocas ocasiones, sin embargo estos reportes han representado problemas significativos. Entre 1948 y 1965 varias cepas de micoplasmas se aislaron del tracto urogenital y tejido sinovial humano (6). De los micoplasmas que se han encontrado en el humano destacan Mycoplasma salivarium, Mycoplasma orale, Mycoplasma buccale, Mycoplasma pneumoniae, Mycoplasma hominis, Mycoplasma genitalium, Mycoplasma pirum, Mycoplasma fermentans, Mycoplasma penetrans, entre otros; tienen como sitios de colonización primario orofaringe y el tracto genitourinario (6). Sin embargo, se ha observado que pueden tener un sitio secundario de colonización como la vagina, cervix, articulaciones artríticas, nódulos linfáticos (7-10), abscesos de tubo ovárico, sangre, riñón y cerebro (11-13), uretra (14), fluido amniótico (15), septicemia neonatal (16), en linfocitos de sangre de pacientes con SIDA y en orina $(17,18)$.

Las especies de flora normal de orofaringe en el humano como $M$. salivarium, $M$. orale, $M$. buccale, $M$. faucium y $M$. lipophilum, generalmente requieren de ambientes anaerobios para aislarlos en laboratorio. M. salivarium usualmente se establece en un 60-80\% en adultos y $M$. orale en un 30-60\% (19). Los escasos reportes de aislamiento de $M$. salivarium de tracto genital bajo en mujeres puede reflejar una transmisión oral-genital $(7,8)$. M. buccale, $M$. faucium y $M$. lipophilum han sido aislados con poca frecuencia de orofaringe. En general, estas cinco especies son consideradas como comensales de humanos, reflejando un papel poco significativo en las enfermedades. Sin embargo, $M$. salivarium cuando se llega a presentar en el tejido gingival puede tener un papel importante en la enfermedad periodontal (19).

\section{Micoplasmas de interés médico.}

Las especies de micoplasmas que frecuentemente se aíslan del humano presentan determinados tejidos o fluidos como sitios primarios de colonización y fuentes nutricionales específicas (cuadro 2).

\section{Mycoplasma pneumoniae}

El agente etiológico de la neumonía por micoplasmas es $M$. pneumoniae, estableciéndose usualmente en orofaringe, bronquios y otras partes del tracto respiratorio superior durante la infección aguda. El microorganismo también ha sido aislado

Vol. 12/No. 4/Octubre-Diciembre, 2001 


\section{JA Rivera-Tapia, ML Cedillo-Ramírez, M Vega-Benítez.}

Cuadro 2

Micoplasmas de interés médico, sitio anatómico primario de colonización y sustrato metabólico que requieren.

\begin{tabular}{lccccc}
\hline & \multicolumn{2}{c}{ Sitio primario de colonización } & \multicolumn{3}{c}{ Sustrato metabólico } \\
Especie & Orofaringe & Tracto genitourinario & Glucosa & Arginina & Urea \\
\hline M. salivarium & + & - & - & + & - \\
M. orale & + & - & - & + & - \\
M. buccale & + & - & - & + & - \\
M. faucium & + & - & - & + & - \\
M. lipophilum & + & - & - & + & - \\
M. pneumoniae & + & - & + & - & - \\
M. hominis & + & + & - & + & - \\
M. spermatophilum & - & + & - & + & - \\
M. genitalium & + & + & + & - & - \\
M. fermentans & + & + & + & + & - \\
M. penetrans & - & + & + & + & - \\
Ureaplasma urealyticum & + & + & - & - & - \\
\hline
\end{tabular}

a partir de pulmón y fluido pleural durante una enfermedad respiratoria aguda $(20,21)$, y del fluido bronquioalveolar de pacientes inmunodeprimidos con neumonía, incluyendo aquellos con síndrome de la inmunodeficiencia adquirida (SIDA) $(22,23)$.

M. pneumoniae se cultiva frecuentemente a partir de muestras de garganta en pacientes convalecientes durante las ocho semanas siguientes a su recuperación clínica. La localización extrapulmonar del microorganismo frecuentemente involucra la diseminación al tejido sinovial posterior a una infección respiratoria o asociado a una artritis séptica con hipogamaglobulinemia $(24,25)$. Además ha sido frecuentemente aislado a partir de lesiones en piel con el síndrome de StevensJohnson/eritema multiforme $(26,27)$, y también se han reportado aislamientos a partir de pacientes con complicaciones neurológicas (28). En dos casos de infección respiratoria se cultivó a partir de muestras de sangre, fluido del pericardio, cerebro y riñón $(12,13)$.

\section{Mycoplasma genitalium}

Tully y col. (29) reportaron el aislamiento de M. genitalium a partir de uretra de dos pacientes homosexuales con uretritis no gonocócica, siendo oportuno señalar que estos datos se presentan con baja frecuencia.

La identificación simultánea de M. genitalium y M. pneumoniae en muestras de garganta de pacientes hospitalizados con enfermedad respiratoria, indican que la garganta puede ser el sitio primario de colonización (30). El hecho de haber identificado a $M$. pneumoniae y $M$. genitalium en fluido sinovial de pacientes con neumonía por micoplasmas y poliartritis, sugiere la necesidad de estandarizar las técnicas para distinguir estos dos microorganismos dentro del tejido del hospedero y en el diagnóstico de laboratorio $(31,32)$, ya que las pruebas moleculares sugieren que $M$. genitalium esta presente en tracto genital de hombres y mujeres $(33,34)$.

\section{Mycoplasma hominis}

La colonización de $M$. hominis en tracto genital y orofaringe ocurre generalmente durante o después del nacimiento, siendo transitoria la colonización en los neonatos, con tendencia a desaparecer después del primero o segundo año de edad $(15,35)$. Dependiendo de la actividad sexual $M$. hominis puede ser nuevamente adquirido en tracto genital bajo $(36,37)$.

El rango de colonización de la cavidad oral de adultos sanos es usualmente menor al 5\%. El microorganismo causa infección neonatal del sistema nervioso central, en piel y en heridas u otros traumas generalmente adquiridos de fuente materna. Este microorganismo además puede estar

\section{Revista Biomédica}




\section{Micoplasmas y su importancia médica.}

asociado con pacientes que han recibido trasplantes y con complicaciones artríticas (38).

Mycoplasma penetrans y Mycoplasma fermentans

Diversas especies de $M$. penetrans han sido aisladas de la orina de pacientes con SIDA, siendo citopatogénico, además de la capacidad para invadir líneas celulares eucariontes $(39,40)$. Por su parte, $M$. fermentans fue aislado por primera vez durante la década de 1950 a partir de tracto genital de hombres y mujeres $(41,42)$, también se ha aislado de diversos tejidos y su aparente diseminación depende de los mecanismos de defensa del hospedero, siendo más vulnerables los pacientes con leucemia, SIDA y durante el tratamiento con drogas inmunosupresoras, pero se ha reportado que también puede ocurrir la diseminación en hospederos con un sistema inmunológico competente (43).

La presencia de $M$. penetrans o $M$. fermentans relacionada con la progresión de enfermedad asociada con el virus de la inmunodeficiencia humana (VIH) generó interés después de observarse que en células mononucleares de sangre periférica, examinadas por medio de la técnica de PCR, 3/46 no progresaron, 3/86 progresaron lentamente y $2 / 27$ progresaron rápidamente cuando M. fermentans estuvo presente. Para M. penetrans se evaluaron 91 muestras, siendo todas negativas. En relación a estos datos, se sugiere que la proporción de pacientes positivos a Mycoplasma fermentans no juega un papel importante en el desarrollo del SIDA en la mayoría de pacientes (44).

En otro caso, el $60 \%$ de pacientes con síndrome de fatiga crónica presentaron infección por diferentes especies de micoplasmas, presentándose $M$. penetrans, $M$ fermentans, $M$. pneumoniae o M. hominis. Los pacientes infectados con más de una especie de micoplasmas generalmente presentaron enfermedades con mayor duración, por tanto se sugiere que existe un incremento en la susceptibilidad a las infecciones por estos microorganismos durante el periodo de la enfermedad (45).

Bauer y col. (46) mostraron que los tejidos renales de pacientes con enfermedades no asociadas al SIDA no revelan la presencia de $M$. fermentans, sin embargo observaron que la presencia de este micoplasma en células parenquimales de riñón de pacientes con SIDA parece estar asociada con nefropatía.

En cinco cepas de M. fermentans se encontró el glicofosfolipido (GLP-III) por medio de anticuerpos específicos, considerándose como un antígeno importante de este micoplasma. Los ensayos inmunológicos con anti-GGPL-III permitieron detectar a este glicofosfolípido en individuos infectados con VIH. La frecuencia de anticuerpos contra GLP-III en pacientes infectados con VIH fue de $44.6 \%$ (29/65), los donadores negativos al VIH presentaron $1.7 \%$ (2/17), y los pacientes con otras enfermedades presentaron $0 \%$ $(0 / 20)$ y $8.7 \%(2 / 23)(47,48)$.

Otras moléculas denominadas lipoproteínas de membrana (MALP-2) de M. fermentans inducen la secreción de interleucina 1-beta y factor de necrosis tumoral en macrófagos de humano, siendo estos eventos cruciales en la expresión de citocinas proinflamatorias (49).

Estudios epidemiológicos predicen que la incidencia de enfermedades por micoplasmas en animales y humanos podría incrementarse, lo cual indica la necesidad de desarrollar nuevas propuestas para la prevención y terapia. Estas propuestas involucran el estudio de la adherencia hacia células hospederas y los mecanismos de evasión contra el sistema inmunológico (50), ya que se conoce que estos micoplasmas presentan características inmunomoduladoras (51).

Hall y col. reportaron para $M$. fermentans la molécula P48 con actividad inmunomoduladora capaz de inducir la diferenciación y apoptosis celular, llegando a ser importante en la evolución de la infección por micoplasmas (52). Por medio de la técnica de PCR se detectó a $M$. fermentans en fluidos de garganta y en muestras de orina en pacientes sanos y en pacientes con

Vol. 12/No. 4/Octubre-Diciembre, 2001 


\section{JA Rivera-Tapia, ML Cedillo-Ramírez, M Vega-Benítez.}

inmunodeficiencia congénita. A partir de 62 muestras de pacientes sanos $11(18 \%)$ fueron positivos en muestras de garganta para este micoplasma, en 46 muestras de orina $8(17 \%)$ resultaron positivas, y de 45 muestras de pacientes, tanto de garganta como de orina $12(27 \%)$ fueron positivos (53). En 20 pacientes que presentaban inmunodeficiencia congénita el $5 \%$ resultó positivo a $M$. fermentans en fluido de garganta y en muestras de orina se observó un $16 \%$, asociándolo a mucosas de personas sanas y en individuos con inmunodeficiencia congénita (53).

La infección por $M$. penetrans y/o $M$. fermentans puede afectar la expresión genética en células humanas, demostrándose así que los cambios inducidos por micoplasmas pueden alterar gradualmente las propiedades biológicas celulares, llegando a producir una forma particular del proceso de la enfermedad (54).

\section{Variación antigénica.}

El potencial adaptativo de las bacterias patógenas es puesto a prueba por los mecanismos de defensa del hospedero, por tanto los microorganismos que pueden responder a su entorno gracias a sus características moleculares adaptativas como son la adherencia, su replicación dentro del hospedero, mimetismo antigénico, la supervivencia dentro de células fagocíticas o la generación de plasticidad fenotípica, tendrán la ventaja de sobrevivir (55). Por su parte, los micoplasmas han desarrollado sistemas genéticos que hacen posible su relación con los tejidos hospederos, básicamente por medio de proteínas de superficie. La variabilidad en la generación de estas proteínas parece estar involucrada con la capacidad para evadir el sistema inmunológico (2, 56), explicando el por qué estos microorganismos son parásitos exitosos, a pesar del reducido tamaño de su genoma.

La adherencia de M. pneumoniae al epitelio respiratorio se considera como un paso esencial en la colonización del tejido y la subsiguiente patogénesis de la enfermedad. Las proteínas de superficie P1 y P30 con pesos de $170 \mathrm{kDa}$ y 30 $\mathrm{kDa}$, respectivamente, han sido identificadas como adhesinas por medio de estudios inmunológicos, bioquímicos y estructurales $(57,58,59)$. Además estas adhesinas inducen respuesta inmunológica en el suero de pacientes en fase de convalecencia y en hámsters infectados experimentalmente (60). Cabe señalar que los anticuerpos generados contra dominios específicos de la adhesina $\mathrm{P} 30$ presentan reacción cruzada con queratina, miosina y fibrinógeno humano (61).

La proteína de superficie P29 confiere diversidad antigénica a $M$. fermentans, presentando un papel importante en lo que respecta a la variación fenotípica. El gen ( $p 29)$ que codifica a la proteína ha sido identificado por medio de la técnica de PCR; siendo localizado en varias cepas de M. fermentans (K-2, SK-5, MY-2, cepa Incognitus) y en cinco cultivos de individuos positivos al VIH (62). La capacidad de variabilidad en la superficie celular contribuye a la supervivencia de $M$. fermentans, particularmente cuando el reconocimiento por parte del hospedero es específico a proteínas de superficie, causando cambios en las reacciones inmunológicas $(62,63)$.

Las bases moleculares de la variación antigénica en diferentes micoplasmas ya han sido descritas $(64,65)$, incluyendo la proteína Vlp de Mycoplasma hyorhinis (66), la proteína V-1 de Mycoplasma pulmonis (67) y la proteína pMGA de Mycoplasma gallisepticum (68), algunas de ellas con importancia médica.

\section{CONCLUSIÓN.}

Los diagnósticos médicos en algunas regiones de nuestro país aún no relacionan a los micoplasmas en diversos episodios infecciosos, de tal forma el estudio que se realiza permitirá conocer más acerca de sus características y así poder tomar en cuenta las ventajas que estas les confieren como moduladores en diferentes procesos celulares y/o cofactores en diversas enfermedades. Por tanto, consideramos que es importante profundizar en el 


\section{Micoplasmas y su importancia médica.}

estudio de ciertos padecimientos en los que están involucrados estos microorganismos y sobre todo proponer tratamientos eficaces, minimizando los riesgos potenciales que puedan generar en la salud.

\section{AGRADECIMIENTOS.}

A la M. en C. Mónica Rosales Pérez, del Laboratorio de Patogenicidad Microbiana del CICM-ICBUAP, por su apoyo brindado en la técnica de micrografía electrónica de transmisión y sus acertados comentarios.

\section{REFERENCIAS.}

1.- Nocard E. Le Microbe de la Peripneumoniae. Ann Inst Pasteur 1880; 12:240-62.

2.- Bové JM. Molecular features of Mollicutes. Clin Infect Dis 1993;17 Supl 1:10-31.

3.- Velleca W, Bird B, Forrester F. Laboratory diagnosis of Mycoplasmas infections. U.S. Department of health and human services public health service. Center for Disease Control, Atlanta, Georgia. U.S.A. 1980; p. 1-5.

4.- Chanock RM. Growth on artificial medium of agent associated with Atypical Pneumonia and its identification as a PPLO. Proc Nat Acad Sci 1962; 48:41-9.

5.- Razin S. Mycoplasma taxonomy and ecology. En J. Maniloff, RN McElhaney, LR Finch and JB Baseman, editores. Mycoplasmas: molecular biology and pathogenesis. American Society for Microbiology, Washington, D.C. 1992. p. 3-22.

6.- Tully JG. Mollicutes: Mycoplasma pneumoniae and Mycoplasma genitalium. Clin Infec Dis 1993; 17 Supl 1:29.

7.- Foy H, Kenny G, Bor E, Hammar S, Hickman R. Prevalence of Mycoplasma hominis and Ureaplasma urealyticum ( $\mathrm{T}$ strain) in urine of adolescents. J Clin Microbiol 1975; 2:226-30.

8.- Gump DW, Horton EL, Phillips CA, Mead PB, Forsyth $B R$. Contraception and cervical colonization with mycoplasmas and infection with cytomegalovirus. Fertil Steril 1975;26:1135-9.

9.- Johnson S, Sidebottom D, Bruckner F, Collins D. Identification of Mycoplasma fermentans in synovial fluid samples from arthritic patients with inflammatory disease.
J Clin Microbiol 2000; 38:90-3.

10.- Jansson E, Hannuksela M, Eklund H, Halme H, Tuuri $\mathrm{S}$. Isolation of a mycoplasma from sarcoid tissue. $\mathbf{J}$ clin Pathol 1972; 25:837-42.

11.- Thomas M, Jones M, Ray S, Andrews B. Mycoplasma pneumoniae in a tubo-ovarian abscess [carta]. Lancet 1975; 2:774-5.

12.- Naftalin JM, Wellisch G, Kahana Z, Diengott D. Mycoplasma pneumoniae septicemia [carta]. JAMA 1974; 228:565

13.- Koletsky RJ, Weinstein AJ. Fulminant Mycoplasma pneumoniae infection. Report of a fatal case, and a review of the literature. Am Rev Respir Dis 1980; 122:491-6.

14.- Tully JG, Taylor-Robinson D, Cole RM, Rose DL. A newly discovered mycoplasma in the urogenital tract. Lancet 1981; 1:1288-91.

15.- Cassel GH, Davis RO, Waites KB, Brown MB, Marriot PA, Stagno S, et al. Isolation of Mycoplasma hominis and Ureaplasma urealyticum from amniotic fluid at 16-20 weeks of gestation: potential effects on outcome of pregnancy. Sex Transm Dis 1983; 10 Supl 4:294-302.

16.- Neman-Simha V, Renaudin H, de Barbeyrac B, Leng JJ, Horovitz J, Dallay D, et al. Isolation of genital mycoplasmas from blood of febrile obstetrical-gynecologic patients and neonates. Scand J Infect Dis 1992; 24:31721.

17.- Montagnier L, Blanchard A. Mycoplasmas as cofactor in infection due HIV. Clin Infect Dis 1993; 17 Supl 1:30915.

18.- Dawson MS, Hayes M, Wang RY, Armstrong D, Kundsin, Lo SC, et al. Detection and isolation of Mycoplasma fermentans from urine of human immunodeficiency virus 1-infected patients. Arch Pathol Lab Med 1993; 117:511-4.

19.- Watanabe T, Matsuura M, Seto K. Enumeration, isolation and species identification of mycoplasmas in saliva sampled from the normal and pathological human oral cavity and antibody response to an oral mycoplasma (Mycoplasma salivarium). J Clin Microbiol 1986; 23:10348.

20.- Nagayama Y, Sakurai N, Tamai K, Niwa A, Yamamoto $\mathrm{K}$. Isolation of Mycoplasma pneumoniae from pleural fluid

Vol. 12/No. 4/Octubre-Diciembre, 2001 


\section{JA Rivera-Tapia, ML Cedillo-Ramírez, M Vega-Benítez.}

and/or cerebrospinal fluid: report of four cases. Scand J Infect Dis 1987; 19:521-4.

21.- Loo VG, Richardson S, Quinn P. Isolation of Mycoplasma pneumoniae from pleural fluid. Diagn Microbiol Infect Dis 1991; 14:443-5.

22.- Lehtomaki K, Kleemola M, Tukianen P, Kantanen ML, Laitinen LA. Isolation of Mycoplasma pneumoniae from bronchoalveolar lavage fluid. J Infect Dis 1987; 155:133941 .

23.- Pérez CR, Leigh MW. Mycoplasma pneumoniae as the causative agent for pneumonia in the immunocompromised host. Chest 1991; 100:860-1.

24.- Taylor-Robinson D, Gumpel JM, Hill A, Swannell AJ. Isolation of Mycoplasma pneumoniae from the synovial fluid of a hypogammaglobulinaemic patient in a survey of patient with inflammatory polyarthritis. Ann Rheum Dis 1978; 37:180-2.

25.- Johnston CLW, Webster ADB, Taylor-Robinson D, Rapaport G, Hughes GRV. Primary late-onset hypogammaglobulinaemia associated with inflammatory polyarthritis and septic arthritis due to Mycoplasma pneumoniae. Ann Rheum Dis 1983; 42:108-10.

26.- Lyell A, Gordon AM, Dick HM, Sommerville RG. Mycoplasmas and erythema multiforme. Lancet 1967; 2:1116-8.

27.- Levy M, Shear NH. Mycoplasma pneumoniae infections and Stevens-Johnson syndrome: report of eight cases and review if the literature. Clin Pediatr 1991; 30:429.

28.- Abramovitz P, Schvartzman P, Harel D, Lis I, Naot Y. Direct invasion of the central nervous system by Mycoplasma pneumoniae: a report of two cases. J Infect Dis $1987 ; 155: 482-7$.

29.- Tully JG, Taylor-Robinson D, Cole RM, Rose DL. A newly discovered mycoplasma in the human urogenital tract. Lancet 1981; 1:1288-91.

30.- Baseman JB, Dallo SF, Tully JG, Rose DL. Isolation and characterization of Mycoplasma pneumoniae strain from the human respiratory tract. J Clin Microbiol 1988; 26:2266-9.

31.- Davis CP, Cochran S, Lisse J, Buck G, DiNuzzo AR, Weber T, et al. Isolation of Mycoplasma pneumoniae from synovial fluid samples in a patient with pneumonia and polyarthritis. Arch Intern Med 1988; 148:969-70.

32.- Tully JG, Baseman JB. Mycoplasma [carta]. Lancet 1991; 337:1296.

33.- de Barbeyrac B, Bernet-Poggi C, Febrer F, Renaudin H, Dupon M, Bebear C. Detection of Mycoplasma pneumoniae and Mycoplasma genitalium by polimerasa chain reaction I clinical samples. Clin Infect Dis 1993: 17 supl 1:83-9.

34.- Taylor-Robinson D, Gilroy CB, Hay PE. The occurrence of Mycoplasma genitalium in different populations and its clinical significance. Clin Infect Dis 1993; 17 supl 1:66-8.

35.- Watts DH, Krohn MA, Hillier SL, Eschenbach DA. The association of occult amniotic fluid infection with gestational age and neonatal outcome among women in preterm labor. Obstet Gynecol 1992; 79:351-7.

36.- McCormack WM, Almeida PC, Bailey PE, Grady EM, Lee Y-H. Sexual activity and vaginal colonization with genital mycoplasma. JAMA 1972; 221:1375-7.

37.- McCormack WM, Lee Y-H, Zinner SH. Sexual experience and urethral colonization with genital mycoplasma: a study in normal men. Ann Intern Med 1973; 78:696-8.

38.- Madooff S, Hooper DC. Nongenitourinary infections caused by Mycoplasma hominis in adults. Rev. Infect Dis 1988; 10:602-13.

39.- Lo S-C, Hayes MM, Wang RY-H, Pierce PF, Kotani H, Shih JW-K. Newly discovered mycoplasma isolated from patients infected with HIV. Lancet 1991; 338:1415-8.

40.- Lo S-C, Hayes MM, Kotani H, Pierce PF, Wear DJ, Newton PB 3d, et al. Adhesion onto and invasion into mammalian cells by Mycoplasma penetrans a newly isolated mycoplasma from patients with AIDS. Mod Pathol 1993; 6:276-80.

41.- Ruiter M, Wentholt HMM. A pleuropneumonia-like organism in primary fusopirochetal gangrene of the penis. J Invest Dermatol 1950; 15:301-4.

42.- Ruiter M, Wentholt HMM. Isolation of a pleuropneumonia-like organism (G-strain) in a case of fusopirillary vulvovaginitis. Acta Derm Venereol 1953; 33:123-9.

\section{Revista Biomédica}




\section{Micoplasmas y su importancia médica.}

43.- Beecham HJ III, Lo SC, Lewis DE, Comer SW, Riley $\mathrm{KJ}$, Oldfield EC III. Recovery from fulminant infection with Mycoplasma fermentans (incognitus strain) in nonimmunocompromised host. Lancet 1991; 338:1014-5.

44.- Ainworth JG, Hourshid S, Easterbrook PJ, Gilory CB, Weber JN, Taylor-Robinson D. Mycoplasma species in rapid and slow HIV progressors. Int J STD AIDS 2000; 11:76-9.

45.- Nasralla M, Haier J, Nicolson GL. Multiple mycoplasmal infections detected in blood of patients with chronic fatigue syndrome and/or fibromyalgia syndrome. Eur J Clin Microbiol Infect Dis 1999; 18:859-65.

46.- Bauer FA, Wear DJ, Angritt P, Lo SC. Mycoplasma fermentans (incognitus strain) infection in the kidney of patients with acquired immunodeficiency syndrome and associated nephropathy: a light microscopic, immunohistochemical, and ultrastructural study. Hum Pathol 1991; 22:63-9.

47.- Li JL, Matsuda K, Takagi M, Yamamoto N. Detection of serum antibodies against phosphocholine-containing aminoglycerolipid specific to Mycoplasma fermentans in HIV-1 infected individuals. J Immunol Methods 1997; 208:103-13.

48.- Matsuda K, Li JL, Ichinose S, Harasawa R, Saito M, Yamamoto N. Monoclonal antibody against Mycoplasma fermentans-specific aminoglycerolipid. Microbiol Immunol 2000; 44:695-702.

49.- Garcia J, Lemercier B, Roman-Roman S, Rawadi G. A Mycoplasma fermentans-derived synthetic lipopeptide induces AP-1 and NK-kappa B activity and cytokine secretion in macrophages via the activation of mitogenactivated protein kinase pathways. J Biol Chem 1998; 273:3491-8.

50.- Rosengarten R, Citti C, Glew M, Lischewski A, Droesse M, Much P, et al. Host-pathogen interactions in mycoplasma pathogenesis: virulence and survival strategies of minimalist prokaryotes. Int J Med Microbiol 2000; 290:15-25.

51.- Rawadi G. Mycoplasma fermentans interaction with monocytes/macrophages: molecular basis. Microbes Infec 2000; 2:955-64.

52.- Hall RE, Agarwal S, Kestler DP. Induction of leukemia cell defferentiaition and apoptosis by recombinant $\mathrm{P} 48$, a modulin derived from Mycoplasma fermentans. Biochem Biophys Res Commun 2000; 269:284-9.

53.- Ainsworth JG, Hourshid S, Webster AD, Gilroy CB, Taylor-Robinson D. Detection of Mycoplasma fermentans in healthy students and patients with congenital immunodeficiency. J Infect 2000; 40:138-40.

54.- Zhang S, Wear DJ, Lo SC. Mycoplasmal infectious alter gene expression in cultured human prostatic and cervical epithelial cells. FEMS Immunol Med Microbiol 2000; 27:43-50.

55.- Razin S, Yogev D, Naot Y. Molecular biology and pathogenicity of mycoplasmas. Microbiol Mol Biol Rev 1998; 62:1096-1156.

56.- Razin S. Adherence of pathogenic mycoplasma to host cells. Biosci Rep 1999; 19:367-72.

57.- Baseman JB, Morrison-Plummer J, Drouillard D, Puleo-Scheppke B, Tyron VV, Holt C. Identification of a 32-kilodalton protein of Mycoplasma pneumoniae associated with hemadsorption. Isr J Med Sci 1987; 23:4749.

58.- Dallo SF, Chavolla A, Basema JB. Characterization of the gene for a 30-kilodalton adhesin-releated protein of Mycoplasma pneumoniae. Infect Immun 1990; 58:41635.

59.- Feldner J, Gobel V, Bredt W. Mycoplasma pneumoniae adhesin localized to tip structure by monoclonal antibody. Nature 1982; 298:765-7.

60.- Morrison-Plummer J, Leith DK, Baseman JB. Biological effects of anti-lipid and anti-protein monoclonal antibodies on Mycoplasma pneumoniae. Infect Immun 1986; 53:398-403.

61.- Dallo SF, Lazzell AL, Chavolla A, Reddy SP, Baseman JB. Biofunctional domains of the Mycoplasma pneumoniae P30 adhesin. Infect Immun 1996; 64:2595-2601.

62.- Theiss PM, Karpas A, Wise KS. Antigenic topology of the P29 surface lipoprotein of Mycoplasma fermentans: differential display of epitopes results in high-frequency phase variation. Infect Immun 1996; 64:1800-9.

63.- Theiss PM, Kim MF, Wise KS. Differential protein expression and surface presentation generates highfrequency antigenic variation in Mycoplasma fermentans. Infect Immun 1993; 61:5123-8.

Vol. 12/No. 4/Octubre-Diciembre, 2001 


\section{JA Rivera-Tapia, ML Cedillo-Ramírez, M Vega-Benítez.}

64.- Dybvig K. DNA rearrangements and phenotypic switching in prokaryotes. Mol Microbiol 1993; 10:465-71. 65.- Wise KS. Adaptive surface variation in mycoplasmas. Trends Microbiol 1993; 1:59-63.

66.- Citti C, Wise KS. Mycoplasma hyorhinis vlp gene transcription: critical role in phase variation and expression of surface lipoproteins. Mol Microbiol 1995; 18:649-60.

67.- Bhugra B, Voelker LR, Zou N, Yu H, Dybvig K. Mechanism of antigenic variation in Mycoplasma pulmonis: interwoven, site-specific DNA inversions. Mol Microbiol 1995; 18:703-14.

68.- Markham PF, Glew D, Whithear KG, Walker ID. Molecular cloning of a member the gene family that encodes pMGA, a hemagglutinin of Mycoplasma gallisepticum. Infect Immun 1993; 61:903-9.

\section{Revista Biomédica}

